\title{
Segregación urbana en la ciudad de Sincelejo, Colombia: caso del barrio San Miguel
}

\author{
Urban Segregation in the City of Sincelejo-Colombia: case of the San Miguel Neighborhood \\ Segregação urbana na cidade de Sincelejo, Colômbia: caso do distrito de San Miguel
}

Rafael Eduardo Hernández Porras. M.Sc. en Proyecto Avanzado de Arquitectura y Ciudad.

rafael.hernandezp@cecar.edu.co

(iD) https://orcid.org/0000-0002-2349-685X

Corporación Universitaria del Caribe, CECAR, Sincelejo, Colombia.

Recibido: Agosto 15 de 2017

Aceptado: Julio 30 de 2018

Publicado: Diciembre de 2018

\section{RESUMEN}

La presente investigación trata el fenómeno de segregación urbana presente en la ciudad de Sincelejo-Colombia, particularmente en el barrio San Miguel. Para este propósito, se han estudiado datos demográficos y sociales que muestran el origen y situación del barrio periférico de la ciudad. Estos datos han sido contrastados con una descripción cualitativa del estado de los equipamientos e infraestructuras urbanas presentes. Posteriormente, se hace una revisión bibliográfica que aborda el tema de la segregación. Con ello, se pretende mostrar que existe un fenómeno de segregación y desigualdad social palpables en San Miguel.

Palabras clave: Segregación Urbana; pobreza; espacio público; desigualdad social; políticas sociales.

\section{ABSTRACT}

The present investigation deals with the phenomenon of urban segregation present in the city of Sincelejo-Colombia, particularly in the San Miguel neighborhood. For this purpose, demographic and social data have been studied, showing the origin and situation of the peripheral neighborhood of the city. These data have been contrasted with a qualitative description of the state of the facilities and urban infrastructures present. Subsequently, a bibliographical review is made that addresses the issue of segregation. With this, it is intended to show that there is a phenomenon of segregation and social inequality palpable in San Miguel.

Keywords: Urban segregation; poverty; public space; social inequality; social policies.

\section{RESUMO}

A presente investigação trata do fenômeno da segregação urbana presente na cidade de Sincelejo-Colômbia, particularmente no bairro de San Miguel. Para tanto, foram estudados dados demográficos e sociais, que mostram a origem e situação do bairro periférico da cidade. Estes dados foram contrastados com uma descrição qualitativa do estado das instalações e infraestruturas urbanas presentes. Posteriormente, é feita uma revisão bibliográfica que aborda a questão da segregação. Com isso, pretende-se mostrar que existe um fenômeno de segregação e desigualdade social palpável em San Miguel.

Palavras-chave: Segregação urbana; pobreza; espaço público; desigualdade social; políticas sociais.

Como citar (APA)

Hernández Porras, R. (2018). Segregación urbana en la ciudad de Sincelejo, Colombia: caso del barrio San Miguel. Procesos Urbanos 5:58-66. DOI: https:// doi.org/10.21892/2422085X.410 


\section{Introducción}

La segregación urbana es un tema que en los últimos años ha sido tocado por diversos autores en Latinoamérica, siendo abordada desde múltiples aspectos, que van desde lo residencial, hasta lo étnico y social. Para Sabatini (2006, p.7), "la segregación residencial corresponde a la aglomeración en el espacio de familias de una misma condición social, más allá de cómo definamos las diferencias sociales".

No podemos limitarnos a desarrollar únicamente un estudio de segregación desde lo residencial, ya que el fenómeno abarca aspectos más amplios. Pérez (2011) menciona que la segregación no es construida solo en términos de diferenciación residencial, sino también en el contacto con lo cotidiano. Lo anterior produce regionalizaciones internas en la ciudad. Por tanto, la segregación pasa a ser un fenómeno con tres dimensiones: residencial (donde la gente vive), territorial (donde la gente realiza sus actividades cotidianas) e interactivo (las relaciones que establecen las redes sociales).

La investigación se centra en el problema de segregación urbana y abandono del barrio San Miguel en la ciudad de Sincelejo-Colombia. Como se mencionó, este es un fenómeno recurrente en muchas ciudades de América latina. En Colombia, se han desarrollado diversos estudios, por ejemplo, la Crítica al Ordenamiento y Control Urbanístico, de Gutiérrez, R. (2014), en la ciudad de Bogotá.

La segregación urbana, ha propiciado un incremento en la vulnerabilidad de los habitantes de San Miguel, llegando, incluso, a fenómenos de violencia y delincuencia común. Los procesos anteriores, estimulan nuevas "formas de diferenciación y vulnerabilidad social, resultantes en crecientes desigualdades sociales, que refuerzan la fragmentación económica y sociocultural" (Veiga y Rivoir, 2009, p.106).

Para estudiar dichos fenómenos, se plantean los siguientes objetivos: escribir los orígenes y características urbanas del barrio San Miguel; definir cómo se presenta el fenómeno de segregación urbana; la relación de la segregación urbana y los fenómenos de exclusión en el barrio, en relación con dichas características.

La hipótesis planteada consiste en la directa relación entre las características urbanas del Barrio San Miguel, sus alrededores y los fenómenos de segregación urbana.

Pese a existir estudios al respecto, no se han encontrado numerosas investigaciones de este tipo en Sincelejo. Las existentes, han tenido un enfoque desde la valuación del suelo. Por lo tanto, el presente trabajo tiene relevancia al describir dicho fenómeno en la ciudad, y puede ser un punto de partida para futuras investigaciones.

\section{Metodología}

El presente es un estudio de carácter cuantitativo, ya que se emplearon datos como estadísticas e índices obtenidos de fuentes como el Departamento Administrativo Nacional de Estadística (DANE). Además, se hace un registro cualitativo de los fenómenos y formas de vida de los habitantes del barrio, así como de los equipamientos e infraestructuras presentes.
Las principales fuentes a tratar son de tipo documental, por tanto, se realizó una revisión de la bibliografía en libros; artículos de revista - principalmente en el campo de las ciencias sociales, específicamente en el área de geografía-; en bases de datos como, Scopus, ScienceDirect, entre otras.

También se emplearon bases de datos del Departamento Nacional de Estadística, cartografía urbana del Plan de Ordenamiento Territorial del municipio de Sincelejo y del Instituto Geográfico Agustín Codazzi.

La búsqueda se realizó filtrando por las temáticas a abordar que fueron: segregación urbana, pobreza y segregación social. Además, aplicando filtros para obtener artículos de libre acceso. Los criterios de análisis y selección de la información fueron: la relevancia y nivel de especialización en la línea de investigación, en este caso relacionada con la segregación urbana; además, la naturaleza de la misma, donde fue de utilidad la información de tipo estadística, que pudo ser contrastada por medio de método de observación directa realizada en el barrio.

\section{Situación y emplazamiento}

La ciudad de Sincelejo se encuentra localizada al noroeste de Colombia, en el Departamento de Sucre, siendo parte de la Región Caribe. Su ubicación le brinda una posición estratégica, ya que es un punto de cruce de dos vías nacionales importantes, que son: la Carretera Troncal de Occidente y la Carretera al Golfo de Morrosquillo.

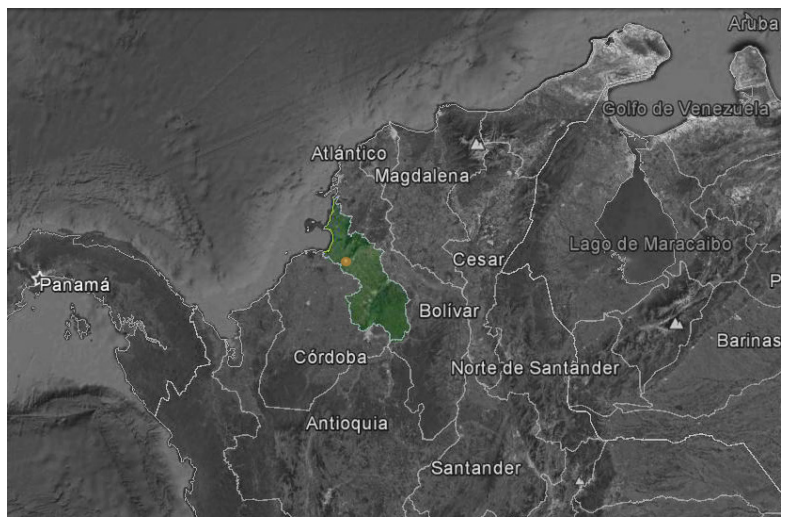

Figura 1. Localización y Límites del Departamento de Sucre y su capital Sincelejo.

Fuente: Editado de google maps.

Esta localización -como ciudad central en la Subregión de Sabanas, de cruce de caminos y posteriores vías nacionales (ver Figura 2) - fue convirtiendo a Sincelejo en un eje comercial y epicentro de constantes migraciones, lo que ha favorecido el crecimiento y expansión de la ciudad, señalados más adelante.

"En 1778 Sincelejo contaba con 1.384 habitantes, en 1870 ya tenía 11.336, y en 1918 el número de población era de 14.722 habitantes" Vilora (2001, p.6).

Estos datos poblacionales de finales del siglo XVIII y principios de siglo XX, contrastan con los recopilados por los censos de los años 1993 y 2005, realizados por el Departamento Nacional de Estadística (DANE), que corresponden a una población de 174.345 habitantes en el año 1993 y 219.305 según el censo de 2005. 


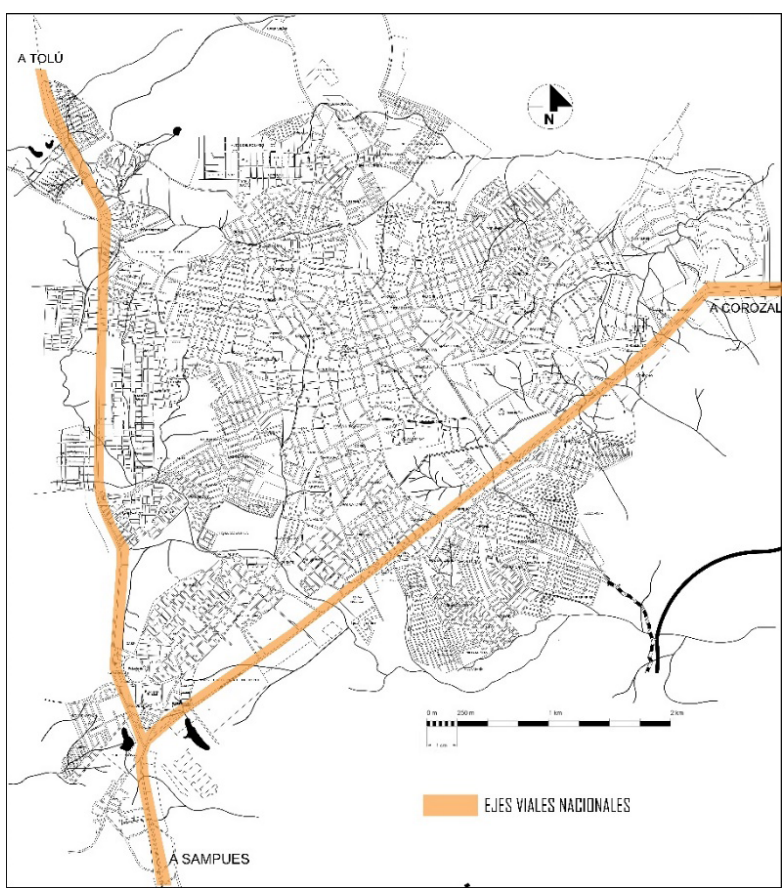

Figura 2. Ejes Viales Nacionales en Sincelejo.

Fuente: Editado a partir del Plan de Ordenamiento Territorial de Sincelejo.

La tasa de crecimiento de la población en la ciudad de Sincelejo es llamativa, según las proyecciones del DANE. A partir del año 1993 hasta el año 2015, creció en más de 100.862 habitantes, lo que correspondería a un $57,8 \%$. Una cifra considerable teniendo en cuenta la capacidad de acogida de la ciudad, relacionada con la infraestructura, vivienda y otros equipamientos urbanos con los que cuenta la ciudad.

Según Naciones Unidas, en 2014 se presentó un crecimiento urbano del $37 \%$ en países con elevado número de habitantes -como India, China y Nigeria-, pese a tener condiciones bastante disimiles. La cifra es bastante cercana al crecimiento presentado en la ciudad de Sincelejo.

Es necesario mencionar que este marcado crecimiento poblacional se debe, en parte, a secuelas del conflicto armando, como el fenómeno de desplazamiento masivo de campesinos desde diferentes zonas rurales de Colombia. Sincelejo - por su centralidad y conexión con estas áreas- se convirtió en un punto de llegada de personas afectadas por la violencia.

Según la Organización de Derechos Humanos Colombia (2005), entre 2002 y 2010 fueron desplazadas: 207.607 personas en 2003, 287.581 en 2004, 310.237 en 2005 y 112.099 en el primer trimestre de 2006, para un total de 917.524 colombianos desplazados. En el caso de Sincelejo, solo en el año 2010 se registraban más de 17312 familias desplazadas, siendo una de las ciudades en Colombia que ha acogido un mayor número de desplazados (EI Universal, 2010).
Tabla 2. Crecimiento Poblacional de Sincelejo desde el año 2000.

\begin{tabular}{cc}
\hline AÑO & POBLACIÓN DE SINCELEJO \\
\hline 2000 & 219.305 \\
2005 & 237.639 \\
2010 & 256.255 \\
2015 & 275.207 \\
2020 & 294.650 \\
$\%$ & $34.5 \%$ \\
\hline
\end{tabular}

Fuente: Departamento Nacional de Estadística DANE.

Se hace necesario realizar este recuento, ya que este aumento poblacional, producto del desplazamiento forzado, ha sido uno de los factores que más ha influido en los fenómenos de segregación urbana en la ciudad; que se ven resaltados, además, por el alto índice de necesidades básicas insatisfechas, que, según el DANE, ronda el $40,41 \%$. Lo anterior se hace más notorio en los barrios de la periferia.

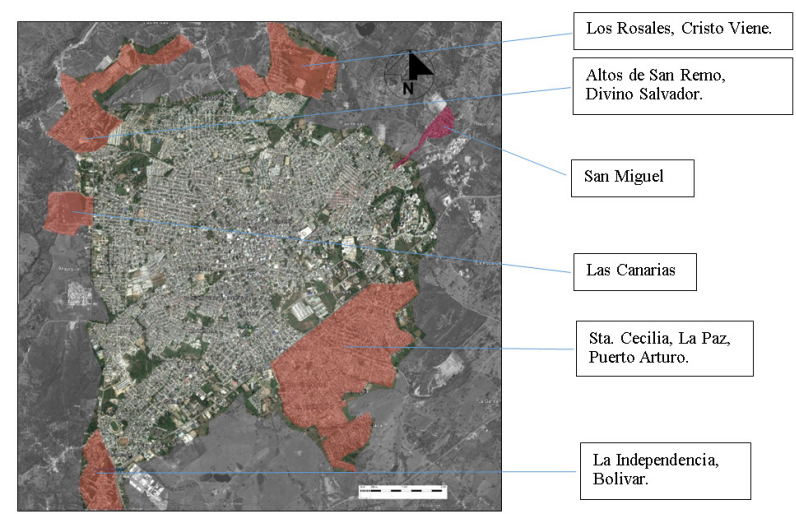

Figura 3. Barrios Periféricos de la Ciudad de Sincelejo.

Fuente: Elaboración Propia a partir de imagen satelital de Google Earth.

Se ha tomado el caso particular del barrio San Miguel, perteneciente a la Comuna 7 Noreste de Sincelejo, ya que encontramos en él algunas características urbanas y sociales encontradas en otros barrios de la periferia. Además, la brecha urbana se hace más notoria, ya que limita al sur con barrios de altos ingresos económicos per cápita y una buena calidad de vida.

En las Figura 4 y 5, se ilustra la división barrial producto de los procesos urbanos iniciados en los años setenta, concretamente el caso de la urbanización Venecia - Primera Etapa, seguido en décadas posteriores por la urbanización Los Alpes. Dichos procesos crearon una barrera física en la ciudad, dividiendo los barrios las Margaritas y, posteriormente, el Barrio San Miguel, que surgió de una división de predios del extinto Instituto Colombiano de Reforma Agraria, con el fin de generar vivienda económica.

Tabla 1. Censo año 1993. Fuente: Departamento Nacional de Estadística DANE.

\begin{tabular}{ccccccccccccccc}
\hline \multirow{2}{*}{$\begin{array}{c}\text { Departamentos } \\
\text { municipios }\end{array}$} & Total & Hombres & Mujeres & Total & Hombres & Mujeres & Total & Hombres & Mujeres \\
\cline { 2 - 8 } & 33.109 .840 & 16.296 .539 & 16.813 .301 & 23.514 .070 & 11.211 .708 & 12.302 .362 & 9.595 .770 & 5.084 .831 & 4.510 .939 \\
Total Nacional & 174.345 & 83.008 & 91.337 & 168.410 & 79.927 & 88.483 & 5.935 & 3.081 & 2.854 & \\
Sincelejo & & &
\end{tabular}

Fuente: Departamento Nacional de Estadística DANE.

60 Procesos 


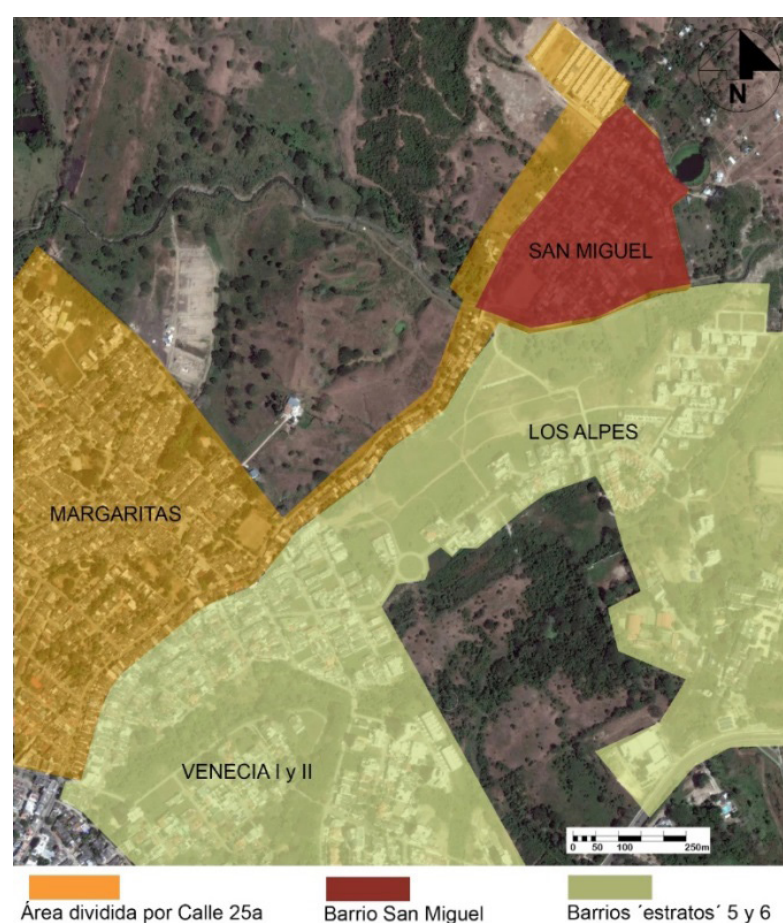

Figura 4. Sector de Estudio.

Fuente: Elaboración Propia a partir de imagen satelital de Google Earth.

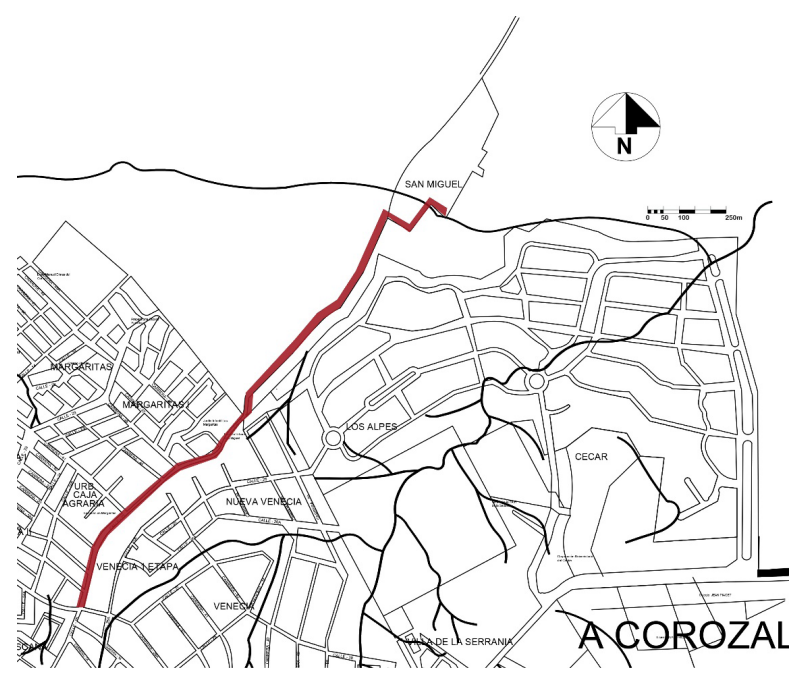

Figura 5. En color rojo Calle $25^{\mathrm{a}}$ y muro posterior que divide el Sector. Fuente: P.O.T Acuerdo agosto 15 de 2000.

La actuación anterior podría relacionarse con lo tratado por Sabatini y Cáceres (2001, p.67), quienes mencionaban que "el tejido socio-espacial ha evolucionado mucho bajo la presión inmobiliaria (la gentrificación, el desarrollo de las colonias cerradas y otros conjuntos residenciales con servicios de seguridad) y el aumento de la delincuencia y de la violencia urbana".

Esta forma de urbanizar, quizá sin quererlo, provocó un hecho segregacionista, en el cual la Secretaría de Planeación Municipal, no les exigió a los urbanistas integrarse, generando un "muro" que se prolonga por 1282 metros, hasta encontrarse con el arroyo Culumuto, que termina de cercar y aislar al barrio San Miguel. Además, hay que mencionar que las recientes actuaciones urbanas han mantenido este mismo esquema. Observamos cómo proyectos de los últimos tres años, se han desarrollado de manera cerrada, lo cual aísla y crea una barrera física (Figura 4).

"Es frecuente que zonas populares colinden con zonas adineradas a la escala de unas manzanas (sea a través de invasiones de tierras o de la construcción de conjuntos cerrados en los intersticios libres de la ciudad" Capron y Gonzáles (2006, p. 67)

Un ejemplo de lo anterior lo encontramos en la urbanización Mirador de los Alpes, al norte del barrio San Miguel, que, siguiendo esta tendencia, se ha proyectado y construido a manera de conjunto residencial cerrado, creando un muro que colinda con San Miguel (Figura 6 y 7 ).

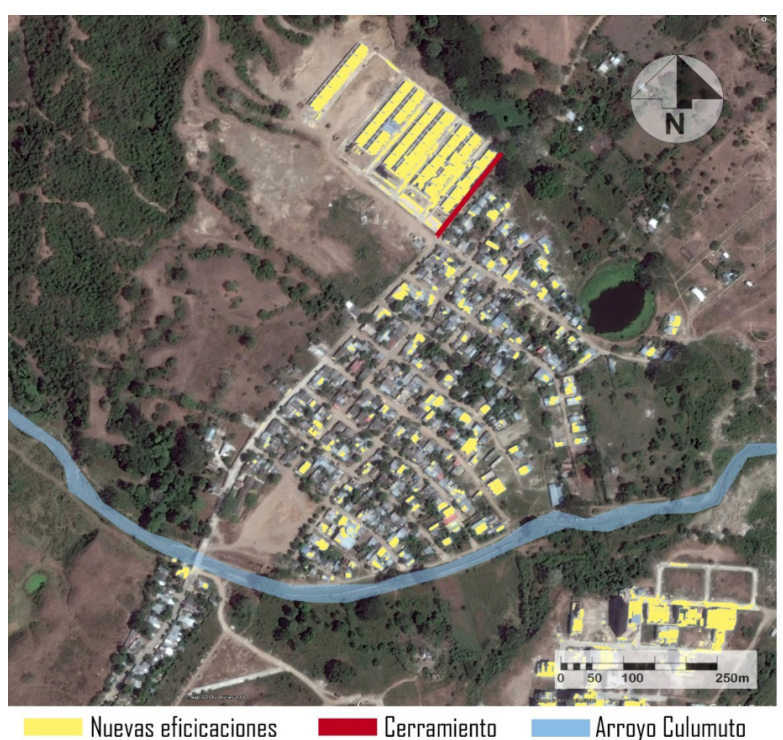

Figura 6. Edificaciones Nuevas en San Miguel, y parte de los Alpes (al Sur). Fuente: Elaboración propia a partir de visita de campo e imagen satelital de google earth.

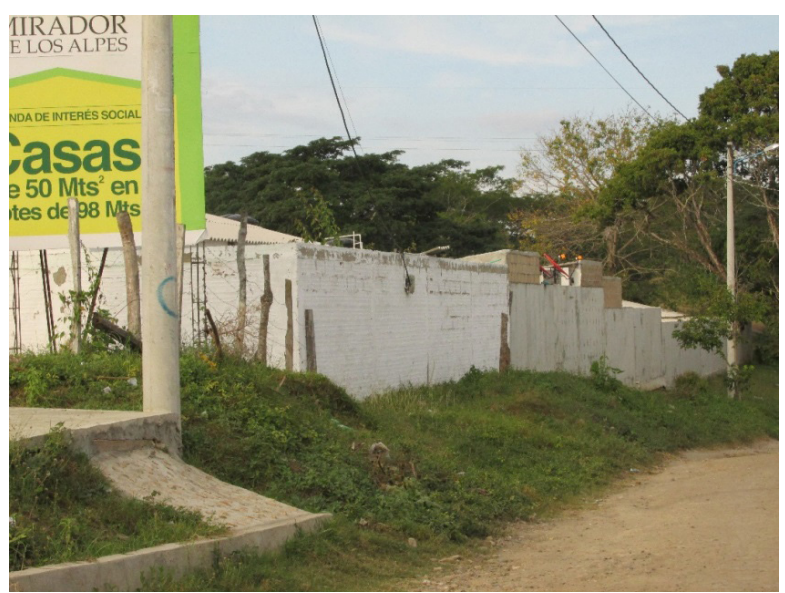

Figura 7. Cerco urbanización Mirador los Alpes, Vivienda de Interés Social. Fuente: el autor. 


\section{Caracteristicas socio ambientales}

Para estudiar el caso de San miguel, se tendrán en cuenta datos de su estructura demográfica, otros datos censales tales como índice de Gini', además de las características habitacionales, de servicios públicos y el espacio público presente, comparándolo con otros barrios de la ciudad de Sincelejo.

\section{Demografía}

La población en San miguel está constituida por personas de origen campesino. Parte de su población, la componen descendientes de la etnia Zenú, que, en estos momentos, se encuentran legalizando la condición de cabildo indígena. Por tanto, no reciben muchos beneficios por ello. En el año 2005, la población se aproximaba a unas 883 personas, de las cuales un 48.3\% eran mujeres. Cohen; (Contreras; Díaz; Martínez; Quiroz; 2005). Cabe resaltar el amplio número de población joven (ver Tabla 3), cifra que ha ido aumentando, en parte por la poca planificación familiar y conocimientos en educación sexual y reproductiva.

Tabla 3. Rango de Edades Población.

\begin{tabular}{cccccc}
\hline Rango de edad (años) & $0-5$ & $6-15$ & $16-30$ & $31-50$ & $51-71$ \\
\hline Número de personas & 115 & 249 & 239 & 204 & 76 \\
\hline
\end{tabular}

Fuente: Cohen et al. (2005).

En 2005, en el estudio realizado por Cohen et al. (2005), se registraron unas 181 viviendas; 158 de ellas con un solo núcleo familiar, 22 con dos familias y 5 viviendas con un núcleo familiar conformado por más de 3 familias. Este dato difiere de la información Catastral del Instituto Geográfico Agustín Codazzi, que tiene un registro de 257 viviendas en la actualidad, pero al no haber un censo actualizado de familias por vivienda, se tomarán los datos y la relación de personas y vivienda de 2005.

Lo anterior nos permite deducir, además, que el núcleo familiar en muchos casos está compuesto por abuelos, padres, hijos, incluso tíos, que viven en una misma unidad de vivienda, llegándose a presentar casos de más de 3 personas por habitación, lo que es considerado hacinamiento. ${ }^{2}$

\section{Economía}

Se presenta, principalmente, una economía de subsistencia. Algunas familias dependen aún de actividades derivadas de su cercanía con la zona rural del municipio; labriegos y jornaleros que trabajan en algunas parcelas cercanas. Existen pequeños establecimientos comerciales, como una llantería de motocicletas, y tiendas de reventa de víveres.

Algunas familias cuentan con miembros que trabajan en algunas actividades del sector de la construcción (ayudantes de obra, ayudantes de electricista, albañiles, etc.); otros se dedican a la venta ambulante y venta de comidas en la calle.

Debido a la informalidad laboral y al bajo grado de escolarización en nivel técnico o superior (menos del $1 \%$ de la población), los ingresos por núcleo familiar, en el mejor de los casos, se encuentran rondando el salario mínimo, que, en Colombia, son unos 273 dólares, con lo cual muchas veces no logran llegar a

$1 \quad$ "El índice de Gini es una de las medidas que más se han utilizado para cuantificar la desigualdad y que también se ha empleado para medir la segregación espacial (Kim y Jargowsky, 2005; Dawkins, 2004)". En Perez, E (2011).

$2 \quad$ "Las Naciones Unidas toman en cuenta la superficie de las habitaciones para sus indicadores de desarrollo sustentable (ONU, 2001), mientras que su división de estadística utiliza la medida de personas por habitación (ONU, 2005)".

62 Procesos final de mes. El gobierno cuenta con el programa de subsidios Familias en Acción, el cual entrega una ayuda de 23 dólares cada dos meses para familias con hijos menores de 6 años, y otra ayuda de 14 a 17.5 dólares mensuales por cada hijo matriculado en la escuela. Los fondos de ayuda muchas veces son irrisorios. Además, encontramos hogares que se encuentran en condición de pobreza extrema, corroborando el censo del Departamento Nacional de Estadística, de 2005.

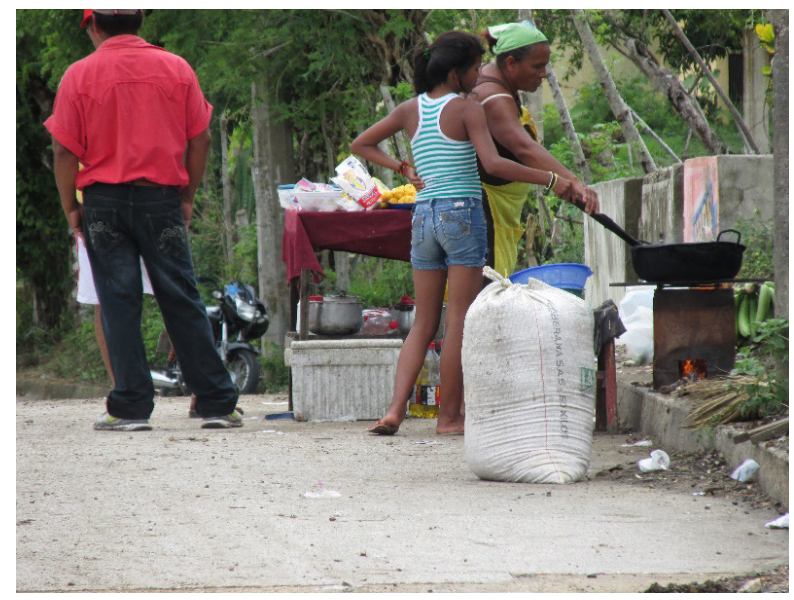

Figura 8. Mujeres trabajando de manera informal para lograr otra fuente de ingresos en el hogar.

Fuente: El autor.

\section{Vivienda y equipamiento urbano}

\section{Vivienda}

La tipología de viviendas del lugar es bastante variable. Va desde algunas construidas con mampostería de bloques de hormigón y cubiertas de teja de asbesto cemento, hasta viviendas de bahareque con cubierta de paja. Otras son construidas con materiales reciclados, similares a las encontradas en chabolas en otros lugares del mundo.

Cabe destacar que la autoconstrucción ha sido la manera como los habitantes de San Miguel han desarrollado sus viviendas. Esto, sumado a los pocos recursos económicos con los que cuentan, ha ocasionado que más del $25 \%$ de las viviendas se encuentren en regular estado y un $74 \%$ en mal estado. Muchas carecen de algunos servicios básicos, como alcantarillado, y están construidas con materiales de baja calidad (piso de tierra, mampostería con sistemas constructivos que no aplican la Norma Sismo Resistente, entre otras (Ver Figura 9, Figura 10).

La anterior cifra es delicada, teniendo en cuenta que la ciudad se encuentra en una zona de amenaza sísmica intermedia, según afirma la Norma Sismo Resistente Colombiana de 2010.

\section{Servicios Públicos}

\section{Agua Potable}

Según el Programa de Gobierno de Sincelejo (2015), en la ciudad de Sincelejo un $78 \%$ de los barrios carecen del servicio de agua potable las 24 horas del día. San Miguel no es la excepción. Por lo tanto, en los días en que no llega el agua potable, se ven en la necesidad de buscarla en un estanque localizado al norte del barrio, o en un pozo profundo que se encuentra en una finca aledaña, siendo este último un predio de tipo privado. 


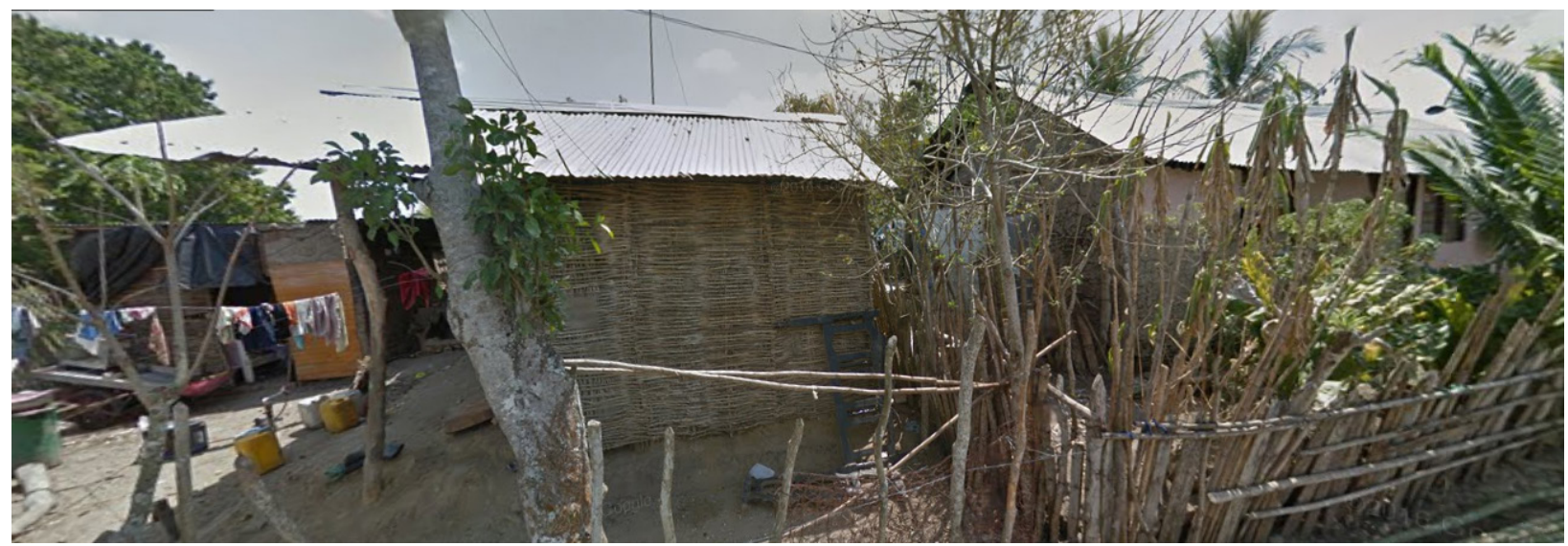

Figura 9. Vivienda en mal estado (pisos de tierra, sin servicio de alcantarillado y agua potable. Fuente: Google Earth.

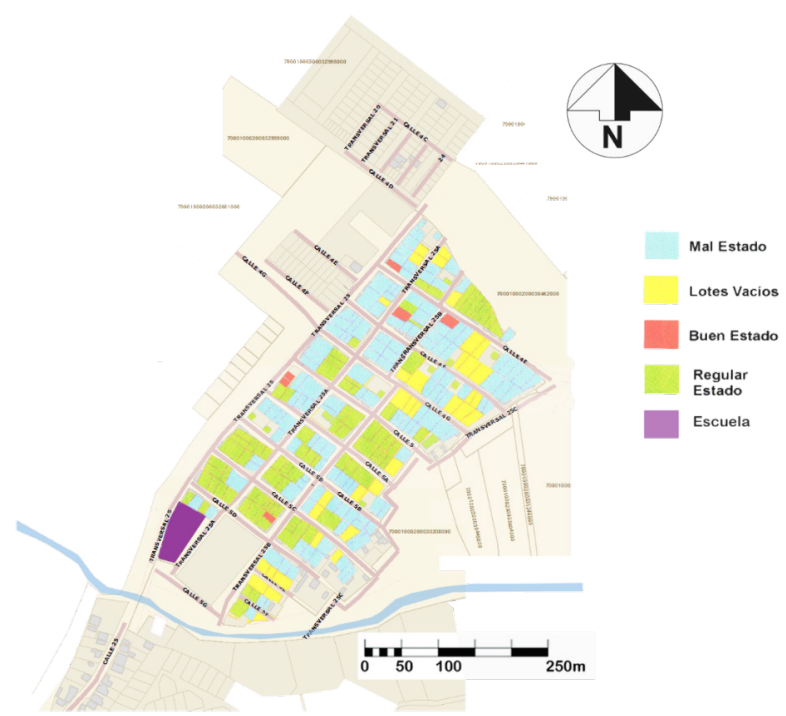

Figura 10. Estado de las viviendas en San Miguel.

Fuente: Elaboración propia con base en estudio de Cohen et al. (2005) contrastado con datos catastrales del Instituto Geográfico Agustín Codazzi (2017) y visita de campo.

\section{Alcantarillado}

La cobertura de la red de alcantarillado en el barrio San Miguel es del $78 \%$, el resto consiste en letrinas. En los hogares más pobres se da deposición a cielo abierto. Cohen et al. (2005)

\section{Gas Natural}

La cobertura de gas es del $68.8 \%$. El resto de los hogares recurren a las hornillas de leña, que se encuentran mal ventiladas, representando un riesgo para la salud de las mujeres que cocinan en ellas.

\section{Energía Eléctrica}

Se presenta una cobertura del $98 \%$, aunque en algunos hogares se conectan de manera irregular a la red. El abastecimiento es por cableado aéreo.

\section{Equipamiento Público}

Salvo por la escuela primaria rural del barrio y la "cancha de futbol" -que no es más que un descampado-, el equipamiento y el espacio público podríamos decir que es nulo. No existe un solo parque, un lugar de recreo para los niños u otro sitio donde las personas puedan tener un sano esparcimiento.

Las vías de acceso, exceptuando la calle 25C, carecen de pavimento. En el mejor de los casos es terreno compactado, lo que ocasiona que, en temporadas de lluvia, se conviertan en verdaderos lodazales, haciendo difícil el desplazamiento de los niños a las escuelas, así como el resto de la población a sus lugares de trabajo (Figura 11).

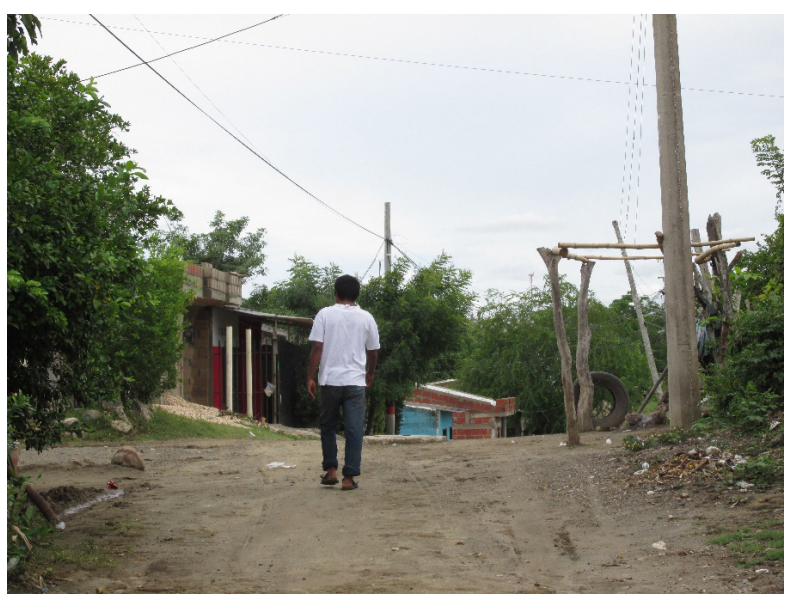

Figura 11. Estado de las Vías.

Fuente: El Autor.

Tampoco existen infraestructuras o sistemas de transporte a las escuelas públicas. Por esto, los chicos se atrasan algunos días en sus actividades académicas, ya que dependen del régimen de lluvias en la ciudad.

La distancia al centro asistencial más cercano es de 3.6 kilómetros, pero "la sola distancia de desplazamiento no explica el esfuerzo que representa para dos individuos con diferentes capacidades de movilidad, y con motivos y destinos de desplazamientos diferentes" Capron y González (2006, p.70). 
La persona acomodada puede tomar su coche y moverse libremente, pero en San Miguel esto es una problemática, ya que la mayoría no posee un coche, ni existe una ruta de transporte público en el barrio, obligando a ancianos, mujeres embarazadas y personas en condición de enfermedad, a transportarse en mototaxis, lo que representa un riesgo para su vida e integridad física.

\section{Zona de amenaza}

Las cotas bajas de terreno aledañas al arroyo Culumuto, han presentado constantes inundaciones anuales, cuando se dan lluvias torrenciales en la ciudad. La mala canalización de los arroyos, así como falta de vegetación y una falta de planeación, hacen que 66 viviendas se encuentren en riesgo, que es mayor para las que están ubicadas a menos de 30 metros del eje del arroyo (Figura 12).

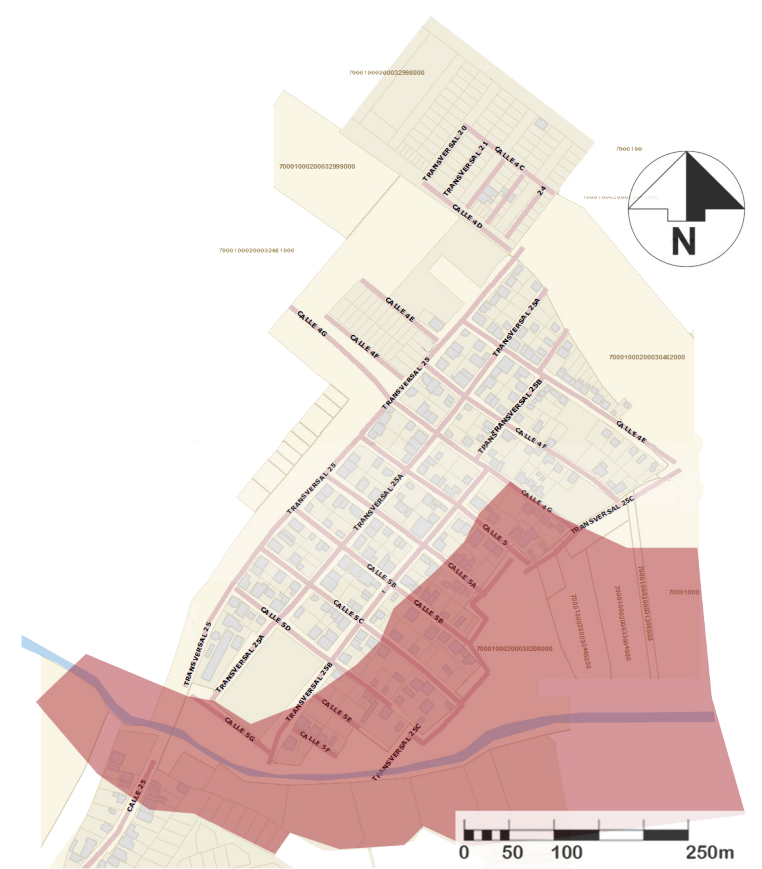

Figura 12. Zona de amenaza de inundación (Teniendo en cuenta la cota más baja).

Fuente: Elaboración a partir de plano topográfico del Instituto Geográfico Agustín Codazzi.

\section{Discusión}

La segregación urbana o segregación espacial, es un fenómeno palpable en diversas ciudades latinoamericanas. Suele entenderse como una división de los grupos sociales en el espacio que conforma la ciudad. Según Pérez (2011, p.406), "Se percibe la segregación como un proceso que separa a la ciudad en unidades delimitadas, cada una de las cuales contiene en su interior una población homogénea que es diferente de la que la rodea".

En el caso de San Miguel, apreciamos núcleos familiares en condiciones de pobreza, que se hacen más notorias debido a las marcadas diferencias socioeconómicas y el sistema de estratificación social establecido en Colombia. Lo anterior, coincide con las tres maneras de abordarla descritas por Grafmeyer.
"En un primer sentido se define como la distribución residencial desigual de la población dentro del espacio que remite a todo un corpus, clásico, sobre la división social del espacio. En un segundo sentido se concibe como el acceso desigual a los servicios y equipamientos urbanos, por lo cual también se refiere a la movilidad de los individuos. En un tercer sentido, la segregación sería la especialización de la distanciación social entre los grupos, en particular en términos de ajustes y conflictos sociales" Capron y González (2006, p.67)

Algunos autores mencionan que la segregación no es un problema, sino un fenómeno, Sabatini (2006) describe que, en algunos casos, se pueden presentar efectos positivos. Explica cómo la formación de enclaves étnicos puede ser benéfica para preservar las culturas y grupos minoritarios, además del enriquecimiento de las ciudades que pueden tornarse más cosmopolitas.

No obstante, del fenómeno segregacionista encontrado en San Miguel, es discutible que existan efectos positivos. Por el contrario, de este se han derivado problemas que han incrementado la pobreza social, o lo que Sabatini (2006, p.8) denomina "nueva pobreza, asociada al surgimiento o reforzamiento de los estigmas territoriales; barrios donde campea el negocio de la droga, la delincuencia, la deserción escolar y el embarazo de adolescentes entre otras formas de desintegración social".

El espacio público, o en este caso la falta del mismo, ha favorecido el detrimento de la calidad de vida y del tejido social. Goycoolea (2006) afirma que la manera de habitar un lugar, los espacios donde se desenvuelven las comunidades y la manera en que se configura, puede generar algunos tipos de actividades o impedir otras. En el caso de San Miguel, la carencia de medios que faciliten la escolarización y reduzcan también la deserción escolar, han ocasionado que algunos jóvenes se vean tentados al mundo de la delincuencia, magnificada y propiciada por un espacio carente de posibilidades para desarrollar un sano esparcimiento, aprendizaje y recreación.

Teniendo en cuenta que "La forma del espacio urbano, así como el modo en que este se gestiona y usa, pueden contribuir a frenar o potenciar ciertos tipos de comportamientos antisociales" (Goycoolea, 2006, p.16.), la ciudad no puede negarle espacios de calidad a algunos sectores y entregarlos a otros. Actualmente, relacionamos la aparición de centros comerciales, como un signo de progreso en la ciudad. Pero algunos autores afirman que son síntomas de la carencia del espacio público. En relación con esto, Capron y González (2006, p.73) comentan que son "lugares de una cohesión social que se debilita, simulacros de espacios públicos donde el acceso está restringido a unos pocos felices".

En relación con la diferencia en la prestación y calidad de los servicios y espacios públicos, con respecto a otras áreas de la ciudad, encontramos que "no solo contribuye a que un lugar sea más concurrido que otro, sino también a generar actitudes de rechazo o pertenencia social" (Goycoolea, 2006, p.15).

Algunos fenómenos de rechazo y estigmatización son notorios. Mientras en San Miguel encontramos una población de ascendencia indígena, en condiciones de pobreza, representantes del "Estrato 1", en los barrios los Alpes y Venecia, vive el estrato 5 y 6 de la ciudad, quienes, además de contar con una mejor situación económica y de ingresos, "en algunos casos 10 veces más altos", también poseen una estructura urbana y 
de equipamientos adecuada. Lo anterior puede ser apreciado como un ejemplo de desigualdad, reflejado, además, en un índice de Gini del 0,474 en Sincelejo.

Esta disparidad, ha producido dos fenómenos; Por un lado, está la auto segregación por parte de las personas de estrato alto, quienes pueden ver a los habitantes de San Miguel como una amenaza, de la cual deben diferenciarse, aislarse, y hasta protegerse. Lo hacen mediante rejas, cámaras, vigilancia privada, creación de unidades cerradas e incluso barrios completamente aislados.

Por otra parte, con el visto bueno de las oficinas de planeación, se han llevado a cabo actuaciones urbanas que han segregado a favor de intereses económicos. Vemos una carencia de vías y espacios que conecten estas dos áreas de la ciudad, lo que además niega cualquier oportunidad de comunicación entre las clases sociales en el espacio público.

"Podemos hablar de segregación cuando existe una intención de discriminación hacia un grupo social que se agrega a una situación de fuertes separaciones sociales en el espacio". Brun (1994) en Capron y González (2006, p.66).

Para los habitantes de San Miguel, ver a diario las condiciones en que viven sus vecinos de altos ingresos y confrontarla con la realidad de su barrio, solo reafirma la sensación de exclusión, de ser diferentes. Con relación a esto, Espino (2008 p.44) mencionaba que "Una vez segregados en barrios homogéneos, los sectores más pobres adquieren estigmas degradantes que dificultan su inserción social y contribuyen a un sentimiento de inferioridad social".

En adición a lo anterior, Healy (2003) afirmaba que la segregación de los pobres en vecindarios donde solo residen personas con las mismas limitaciones agudiza su marginación, convirtiéndose en "áreas de desventajas concentradas". Lo anterior obliga a pensar en la necesidad de espacios públicos que vinculen los barrios de mayores ingresos, en este caso los Alpes y Venecia, con los sectores pobres (San Miguel). Que sean estos espacios, lugares que propicien un dialogo y conocimiento mutuo. Lo anterior, iría acorde con el planteamiento de Espino (2008), para quien es mejor que la interacción entre los diferentes grupos se dé en áreas neutrales, como el lugar de trabajo o el espacio público.
Para Capron y González (2006), las estrategias de movilidad, la accesibilidad y las redes sociales que se tejen, marcan la capacidad de las personas y los lugares para vincularse y acceder a la ciudad. Este hecho, puede significar estar o no segregado. En el caso de San Miguel, varias de las características del barrio coinciden con lo primero.

\section{Conclusión}

No se puede seguir legitimando la segregación como un modelo de desarrollo urbano, ya que solo incrementa los problemas que padecen las personas más pobres en la ciudad. La segregación es, en parte, resultado de la desigualdad social, pero, a la vez, de políticas que han desfavorecido las posibilidades de integración.

Al ser la segregación urbana un fenómeno con marcada incidencia social, el mismo debe ser tratado con políticas impulsadas por las instituciones de gobierno, debido a que resulta difícil realizarlas desde la empresa privada, que busca un beneficio económico, dejando de lado, en muchos casos, el beneficio social. Es por esto que los gobiernos deben mediar y construir políticas de planeación y desarrollo que procuren disminuir o mitigar las condiciones que favorecen la segregación urbana.

En ocasiones, tratando de actuar en el marco de estas políticas, se han realizado intervenciones para mejorar las condiciones de vivienda. Esto, por sí solo, no genera un cambio profundo y no soluciona el problema de segregación vivido en la ciudad. Lo anterior, ha quedado demostrado en el mundo, con fallidas políticas de vivienda social, donde solo se ha pensado en el objeto vivienda, ignorando los componentes urbanos y sociales alrededor de la misma.

Ya que la interacción de los barrios, se da entre habitantes que provienen de grupos sociales con diferente formación y capacitación, es difícil que coincidan en lugares privados, esto le da al espacio público una posición privilegiada, tal vez la más importante para permitir la interacción entre ellos. Es, en este espacio, donde se pueden generar nuevas relaciones sociales que permitan remover algunos estigmas creados.

\section{Referencias}

Capron, G y González, S. (2006). Las escalas de la segregación y de la fragmentación urbana. Travaux et Recherches dans les Amériques du Centre. Centro de Estudios Mexicanos y Centroamericanos Distrito Federal. Mexico. Retrieved from: http://www.redalyc.org/ articulo.oa?id $=423839505006$

Cohen, E; Contreras, W; Díaz, J; Martínez, F; Quiroz, S. (2005). San Miguel, aldea Urbana Ecosostenible. Corporación Universitaria del Caribe CECAR. Sincelejo. Colombia.

Correa, J y Lozano, R. (2013). Si usted gana $\$ 2.000 .000$ es rico para el banco mundial. Editorial el tiempo. Bogotá DC. Retrieved from: http://www.eltiempo.com/archivo/documento/CMS-12779552

Comisión asesora permanente para el régimen de construcciones sismo resistentes. (2010). Reglamento Colombiano para construcción sismo resistente NSR-10. Ministerio de ambiente, vivienda y desarrollo territorial. Colombia

Departamento nacional de Estadística. (2015). Anexo pobreza monetaria y multidimensional. Presidencia de la republica de Colombia. Retrieved from: https://www.dane.gov.co/index.php/estadisticas-por-tema/pobreza-y-condiciones-de-vida/pobreza-y-desigualdad/ pobreza-monetaria-y-multidimensional-en-colombia-2015 
Espino, A. (2008). La segregación urbana: Una breve revisión teórica para urbanistas. Revista de Arquitectura, Universidad Católica de Colombia. Bogotá DC. Retrieved from: http://www.redalyc.org/pdf/1251/125112541006.pdf

Healy, P. (2003). "Institutionalist theory, social exclusión and governance". Social Exclusion in European Cities: Processs, Experiences, and Responses, edited by Ali Madanipour, Göran Cars, and Judith Allen. London: Routledge. p.53. En Espino, A. (2008). La segregación urbana: Una breve revisión teórica para urbanistas. Revista de Arquitectura, Universidad Católica de Colombia. Bogotá DC. Retrieved from: http://www.redalyc.org/pdf/1251/125112541006.pdf

Goycoolea, R. (2006). Violencia y espacio urbano. Quórum. Revista de pensamiento iberoamericano, núm. 16. Universidad de Alcalá. España. Retrieved from: http://www.redalyc.org/pdf/520/52001602.pdf

Gutiérrez, R. (2014). Segregación urbana en Bogotá. Crítica al ordenamiento y control urbanístico en materia de construcción. Cuadernos de vivienda y urbanismo. Universidad Javeriana. Bogotá. Colombia. Retrieved from: http://revistas.javeriana.edu.co/index.php/ cvyu/article/view/8750

Naciones Unidas. (2014). Más de la mitad de la población vive en áreas urbanas y seguirá creciendo. Departamento de asuntos económicos y sociales. Retrieved from: http://www.un.org/es/development/desa/news/population/world-urbanizationprospects-2014.htm

Organización de Derechos Humanos Colombia. (2005). Resultados del Censo general de Colombia DANE 2005. Retrieved from: http:// www.ddhh-colombia.org/html/CENSOGENERALDEL2005.pdf

Pérez, E. (2011). Segregación socioespacial urbana. Debates contemporáneos e implicaciones para las ciudades Mexicanas. Estudios Demográficos y Urbanos. El Colegio de México, A.C. Distrito Federal, México. Retrieved from: http://www.redalyc.org/articulo. oa?id=31223581006

Quessep, J. (2015). Programa de gobierno, “Unidos por el progreso". Municipio de Sincelejo. Retrieved $\quad$ from: $\quad \underline{\mathrm{ht} t \mathrm{p}: / /}$ sincelejo-sucre.gov.co/apc-aa files/61383166366532633430663865366465/unidos-por-el-progreso-jacobo-quessep-espinosa. pdf

Sabatini, F. (2006). La segregación social del espacio en las ciudades de América Latina. Banco interamericano de Desarrollo. Departamento de Desarrollo Sostenible. División de Programas Sociales. Retrieved from: https://publications.iadb.org/ handle/11319/5324

Sabatini, Fy Cáceres, G. (2001). Segregación residencial en las principales ciudades chilenas: Tendencias de las tres últimas décadas y posibles cursos de acción; En Capron, G; González, S. (2006). Las escalas de la segregación y de la fragmentación Urbana Trace. Travaux et Recherches dans les Amériques du Centre.

Veiga, D y Rivoir, A. (2009). Fragmentación socioeconómica y segregación urbana en Montevideo. Revista de Ciencias Sociales. Departamento de Sociologia. Retrieved from: http://uah.summon.serialssolutions. com/2.0.0/link/0/eLvHCXMwnV3NSsQwEB4WQRBEFBV IS Q3f5s7BZWZNUtgq4nQfBSpkla66GRdvfgc0j7Is50x8Qj3tNSIZJyMw3yXwTgMAfus4 mxBpSQdJo0IvxEyRm5cTrbROszRQqcu84Zf34HUeLB6j2QD6T uYOOEROr sVoX9waGpR3xZSSqNLBaOUHfK6iK3t2OCyX5ji 8 IhcWpf PzRVOCMOkdKZp sdpP95eTEh3DQAT8xa3fqCAamPoZ7wo15k76tis26FLxUlkPUzZpp_UplihMKRZcVlopdPYEpvH87eHJ6QQn3239ilQrOvdtvWaJ qRNCsI37IPtDwOEU9pGT3MtIQ4bTZyCiMapQ3mh3opgiimnmemhCN9SZCgPPPwe5layLLcddwl77uMI3Elews6xW5pp8NH7$\underline{\operatorname{AgJ} 20 S 4}$

Vilora, J. (2001). Cuadernos de historia económica y empresarial; ganaderos y comerciantes en Sincelejo, 1880-1920. Banco de la República de Colombia. Retrieved from: http://www.banrep.gov.co/documentos/publicaciones/regional/cuadernos/8.pdf

Red de Bibliotecas virtuales de ciencias sociales de América Latina y el Caribe. http://biblioteca.clacso.edu.ar/gsdl/collect/clacso/ index/assoc/D9393.dir/h.pdf

El Universal. (2010). Sincelejo, hogar de desplazados. Cartagena. Colombia. Retrieved from: http://www.eluniversal.com.co/sincelejo/ local/sincelejo-hogar-de-desplazados 